\title{
Influences on pattern formation during non-isothermal phase separation in local and nonlocal phase-field models
}

\author{
Thomas Stephens \\ Faculty Mentor: Dr. Thomas Wanner \\ Undergraduate Research in Computational Mathematics \\ George Mason University, Fairfax, Virginia
}

\begin{abstract}
The classical phase-field model represents a coupling of an Allen-Cahn type nonlinear equation with a standard diffusion equation and has been proposed to describe uniform phase separation in a pure substance. This research considers an extension of that model which incorporates a more accurate approximation to the diffuse interface between states of matter through the use of a nonlocal operator. Results of simulations under this model are compared with those of the classical model in order to understand the effects of the nonlocal contribution. Attention has been given to the behavior of the underlying temperature field during early phase separation. We use the tools of computational homology to quantitatively compare patterns in the phase field under both models. Simulations show that the complicated patterns in the phase field persist longer during the solidification process in the nonlocal extension of the classical model.
\end{abstract}

\section{Introduction}

An interesting phenomenon occurs when a sample of some material containing more than a single phase of matter is allowed to progress toward thermodynamic equilibrium. For example, when watching the individual pieces of crushed ice in a glass of water over time we notice that the solid ice tends to either fuse with nearby chunks of ice or simply melt away into the surrounding liquid water. When considering an entire sample starting from some initial mixture of phases, the progress toward thermodynamic equilibrium is known as phase separation. We will use the term pattern to refer to the distribution of regions of solid and liquid throughout a sample, and we concern ourselves with how this pattern evolves and when we may assume it reaches equilibrium (or fails to change over time). With what turns out to have an important effect on the driving force, we take into account temperature fluctuations throughout our sample, making our simulations of this process non-isothermal. We are interested in this problem for several reasons. First, physical properties of manufactured materials will depend in some degree on the final distribution of the applicable phases of matter throughout a sample. Understanding mathematical models describing phase separation phenomena are then essential to predicting desired physical properties. This leads directly to another challenge, that of being able to make some sort of quantitative assertion about two non-identical patterns of phase distribution being essentially equivalent. Making this measurement is fundamentally a problem of geometry. The most intrigue however may lie in the numerical study of the systems of partial differential equations that have been used to model this phenomenon. While not addressed here there are numerous interesting issues that arise in these types of models, 
such as stochastic terms used to simulate noise, and stability issues regarding our proposed parameters.

The transition toward thermodynamic equilibrium manifests itself as some thermodynamically advantageous redistribution of solid and liquid regions throughout the sample. We will see that this evolution tends to organize and simplify the pattern of an initially well-mixed sample. (Figures 4 and 2 may provide some sense of what can be expected.) The thermodynamic description and the mathematical modeling technique used here originate in the foundational work of Cahn and Hilliard [4, 2], and closely follows the methods of the recent works of Hartley and Wanner [8,9]. Specifically, we consider a thermodynamic description that allows for the formulation of a free energy functional. Our model accounts for contributions to the total free energy arising from the existence of solid-liquid interfaces, as well as from local temperature fluctuations resulting from the solidification process itself (see Equations (2) and (3), fuller descriptions of each term in these equations follows). The strategy used to describe the evolution of this process begins by following the solutions that minimize the free energy functional. We pursue two different approaches to capturing the role of the interfacial energy in the description of the total free energy of the system and compare their effects on pattern formation.

We will give particular attention to the structure of the pattern as areas in the domain rapidly change phase early in the simulations, as well as to the pattern resulting from the models run to near equilibrium. The influence of the temperature field on the evolution of the phase will also be discussed.

\section{Phase-Field Modeling}

A theory developed in the foundational work of Cahn and Hilliard in the late fifties and early sixties describes the interface between two phases of matter as being diffuse, or essentially ill-defined in a way suggesting that a continuum exists between distinctly solid and distinctly liquid phases of matter (see $[2,3,4]$ ). The width of this interface is postulated to be on the order of magnitude of the width of several crystal lattice planes. Also, this perspective is consistent with that of van der Waals, dating back to 1893 [2].

To describe the state of matter as we move through some distance, for example across an interface between solid and liquid regions, we can define a continuous function taking on values that would indicate if the material is solid, liquid, or somewhat in between. We label this function $\phi$, and in this report we interpret values of $\phi$ near -1 to represent the solid phase of matter and values near +1 to indicate the liquid phase, and can expect $\phi$ to take values between -1 and +1 through an interface between solid and liquid regions occurring in our sample. When considered over our entire spatial domain (the unit square $\Omega \subseteq \mathbb{R}^{2}$ ), for a fixed $t, \phi(t, x)$ provides a snapshot of the distribution of the phases of matter within our sample (See Figure 4). As we are interested in the evolution of the pattern encoded by $\phi$, we let $t$ represent time and will consider the phenomenon as it proceeds from some initial condition $\phi(t=0, x)=\phi_{0}$.

The phase-field models we consider are non-isothermal, meaning that the temperature throughout the domain of the sample will be allowed fluctuate over time. The description of the temperature will be captured in the function $u(t, x)$, where the value of $u$ should be interpreted as measuring how far above or below the melting temperature the ambient temperature field at $x$ happens to be (for some fixed $t$ ). In other words as some matter at $x$ melts, it must draw energy from its surroundings, this scenario will be indicated by a drop in the value of $u$. 
Temperature has an influence on the melting or solidification of any material, and so it is that $\phi$ and $u$ are coupled in the phase-field models under consideration. As thermal energy is transfered to a material it may melt, and a further transfer of heat may cause the liquid to change to gas. (We are concerned only with transitions between solid and liquid, and therefore only focus on behavior around the melting point. The technique being described here can account for more sophisticated considerations, for a discussion of more general phase-field models see [1].) Thus, considering some material at a point $x_{0}$ in our sample existing at some phase, for example $\phi\left(x_{0}\right) \simeq-1$ : indicating solid, and learning that matter is absorbing thermal energy (indicated by $u_{t}\left(x_{0}\right)<0$ ), it should be thermodynamically advantageous for that matter to begin to melt. If this were allowed to proceed we should expect that $\phi_{t}\left(t, x_{0}\right)>0$, i.e. that the value indicating its phase of matter is increasing toward the value of +1 , and the matter at $x_{0}$ may become liquid. Describing the behavior over all possible cases is handled by a double-well potential energy functional $F(\phi, u)$ that accounts for both the phase and the underlying temperature field. This functional is depicted in Figure 1 where the combined state $(\phi(x), u(x))$ is mapped to a measure of potential energy. Moving toward thermodynamic equilibrium causes this state to change in time in such a way as to be drawn toward the minimum value of the potential energy functional. This construction provides the mechanism for our models to change the distribution of solid and liquid regions throughout the sample. (The figure shows the existence of a potential energy barrier between the solid and liquid phases for a given temperature. This barrier represents the energy inherent in the transition from one phase of matter to another.)

With $\phi(t, x), u(t, x)$, and $F(\phi, u)$, we have assembled the pieces needed to construct a phase-field model. For a fixed time we can look at $\phi(t, x)$ to see how the phase varies throughout the spatial domain, obtaining a snapshot of the pattern. Looking at $u(t, x)$ we obtain a thermal picture of the domain, where the values of $u$ will tell us whether a particular region is above or below its melting temperature. We can also vary the time parameter to gather this data as the phenomenon unfolds. The double-well potential function $F$ couples the temperature with the state of matter in a way that is consistent with physical reality. A phase-field model considers the free energy implied by these phenomenological descriptions of our system. The existence of interfaces between solid and liquid boundaries supply a contribution to the free energy on our domain, as well as does the temperature captured by the function $u$. By having constructed these functions to be continuous in space and time, a phase-field model is able to compute the free energy associated with a snapshot of these two field variables in time and evolve to minimize this energy.

\section{Proposed Models}

It is the method of interrogation of the phase-field variable that distinguishes our two models. What we are looking for in each is a determination of whether an interface exists in some region, as well as an indication of how sharp an interface between two phases is. Sharpness refers to the thickness of an interface, in the instance where solid and liquid regions are separated by a distance of relatively few lattice planes the interface is said to be sharp. This information will allow us to account for the free energy inherent in such interfaces, and include this in the total free energy of the system. As originally proposed by van der Waals, and addressed specifically in $[8,9]$, the contribution to the free energy at any point on the domain ought to consider not only the state of the immediate surroundings, but should also consider the composition of the material in some wider neighborhood 

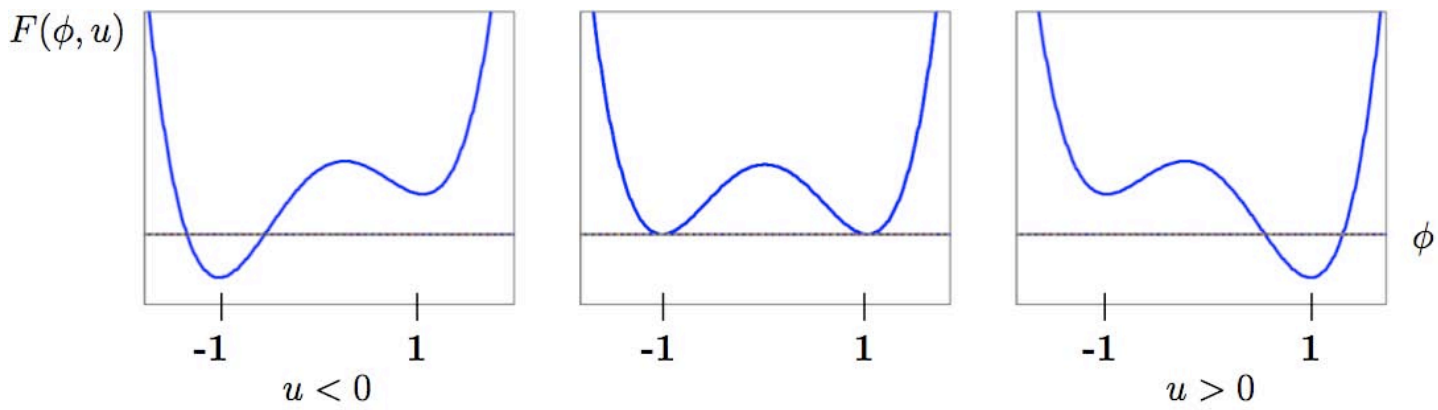

Figure 1: Double well potential function $F(\phi, u)$. The variable $\phi$ is taken along the abscissa, and the value of $u$ taken at the point under evaluation affects the tendency to solidify or to melt. Minimization of the free energy functionals in (2) and (3) acts to draw the value of $\phi$ toward the minima of $F$. These minima are dependent on $u$, and in general remain near -1 and +1 .

of the point under evaluation. This perspective suggests the use of a nonlocal operator to interrogate $\phi$ on the domain. In the investigations presented here, the influence of the surrounding material is determined by the radially symmetric weighting function $J$, expressed by

$$
J(x)=\frac{1}{\varepsilon^{2}} e^{-\left(x_{1}^{2}+x_{2}^{2}\right) / 2 \sigma^{2}},
$$

where $\sigma$ scales the footprint of the weighting function, and is fixed.

Computing the convolution of $J$ and $\phi$ captures the interfacial free energy present at any point. The model that captures the evolution as determined by this method will be referred to as the nonlocal extension to the classical phase-field model, shown by Eqn. (2).

Alternatively, and consistent with the numerical investigations predominant in the literature, the phase-field variable could be interrogated using the gradient operator. This can be viewed as a first-order approximation to the radially symmetric nonlocal operator $J$. As an expression on the partial derivatives of $\phi$, the gradient reveals the sharpness of an interface, should it come across one, by associating points in the domain having liquid on one side and solid on another. The gradient of the phase-field variable will then be large where interfaces are sharp, and including this value in the total free energy of the system will have the effect of penalizing such interfaces. Thus we will see that this model tends to minimize the sharpness of interfaces, and to minimize the total length (or area, in three dimensions) of all interfaces. The evolution equations derived while using the gradient operator will be referred to as the local or classical phase-field model and is shown in (3).

The free energy functionals described above take the following forms:

\section{Nonlocal free energy}

$$
E[\phi, u]=\frac{\varepsilon^{2}}{2} \iint_{\Omega} J(x-y)(\phi(x)-\phi(y))^{2} d x d y+\int_{\Omega} F(\phi, u) d x
$$

\section{Local free energy}

$$
E[\phi, u]=\frac{\varepsilon^{2}}{2} \int_{\Omega}|\nabla \phi|^{2} d x+\int_{\Omega} F(\phi, u) d x
$$


Both models incorporate the same potential function $F(\phi, u)=\frac{1}{4}\left(\phi^{2}-1\right)^{2}-u \phi$ which is used to drive $\phi$ toward the minima of $F$, which lie near -1 and +1 . This potential is a function of both temperature and phase and takes the form of a double well, corresponding to the two permissible states of matter that we are considering. The middle hump of the double-well corresponds to the energy required to transform the material from one phase to another, a property that is specific to the material being modeled (see Figure 1, also this interpretation of the energy barrier comes from a footnote in [3]).

The systems of coupled evolution equations that describe the minimization of the functionals (2) and (3) appear as:

\section{Nonlocal Model}

$$
\begin{aligned}
u_{t} & =\Delta u-\lambda \phi_{t}, \\
\varepsilon^{2} \phi_{t} & =J * \phi-c \phi+f(\phi, u)
\end{aligned}
$$

\section{Local Model}

$$
\begin{aligned}
u_{t} & =\Delta u-\lambda \phi_{t}, \\
\varepsilon^{2} \phi_{t} & =\varepsilon^{2} \Delta \phi+f(\phi, u)
\end{aligned}
$$

We see that the temperature field evolves according to a standard diffusion equation with the local change in the phase as a source term. The constant parameter $\lambda>0$ corresponds to the latent heat specific to the material being modeled, providing a link betweeen specific material properties and the energy required for a phase change. In the local model we arrive at the non-isothermal Allen-Cahn equation describing the evolution of the phase field. The nonlinearity

$$
f(\phi, u)=-\frac{\partial F}{\partial \phi}=-\phi\left(\phi^{2}-1\right)+u
$$

acts to drive the value of $\phi$ toward the minima of $F$ as it appears in Figure 1. Alternatively, the minimization of the nonlocal model arrives at $J * \phi-c \phi$ as the method of capturing the interfacial free energy. This is where the weighting of a collection of values for $\phi$ takes place. The parameter $c$ is dependent upon $J(x)$, and is chosen to maintain the constraint corresponding to $\int \Delta \phi d x=0$ as it exists in the local model, thus we have the requirement that $\int J * \phi-c \phi d x=0$. In other words, $c$ is chosen to be the average of $J$ on $\omega$. In both free energy formulations (2) and (3), the parameter $\varepsilon$ can be interpreted as a domain scaling constant or as representing the mean free path associated with the molecular composition of the material. Careful derivations of these systems can be found in [8] and in the work of Fife [6], along with a more detailed discussion of the physical parameters that are specific to the material being modeled. Our simulations were conducted using the parameter values $\varepsilon=0.005$, and $\lambda=0.5$.

The system will be considered on the two-dimensional square, $[0,1] \times[0,1] \subseteq \mathbb{R}^{2}$ with spatially periodic boundary conditions. The solutions then will satisfy

$$
\begin{aligned}
& u\left(t, x_{1}, x_{2}\right)=u\left(t, x_{1}+1, x_{2}\right)=u\left(t, x_{1}, x_{2}+1\right), \quad \text { and } \\
& \phi\left(t, x_{1}, x_{2}\right)=\phi\left(t, x_{1}+1, x_{2}\right)=\phi\left(t, x_{1}, x_{2}+1\right) .
\end{aligned}
$$

A representative of the long term results of our numerical investigations are shown in Figure 2, where the local model (5) achieves absolute minimal lengths of its interfaces, 


\section{Local Model:}
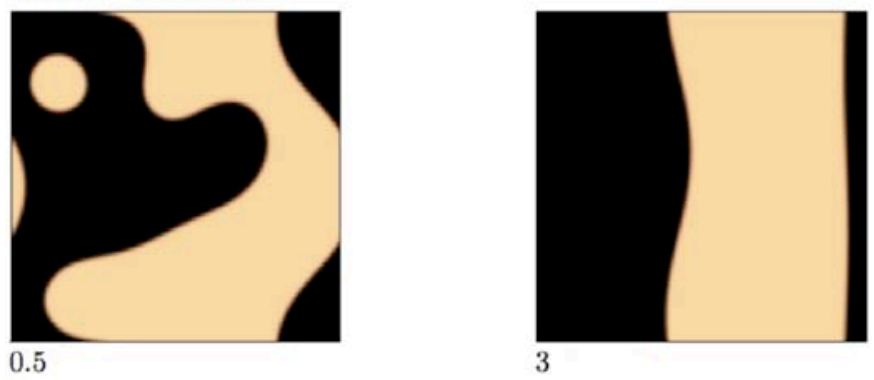

time

\section{Nonlocal Model:}

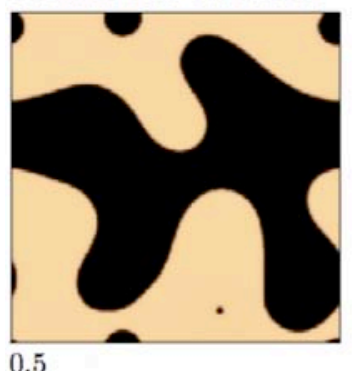

0.5

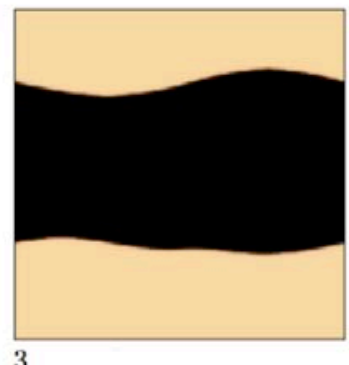

3

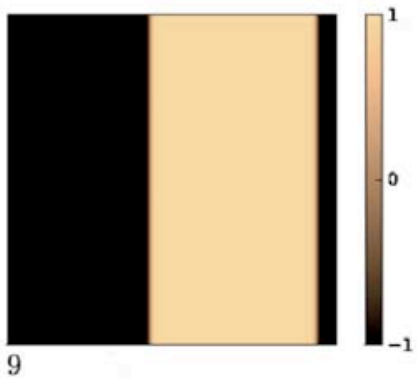

9

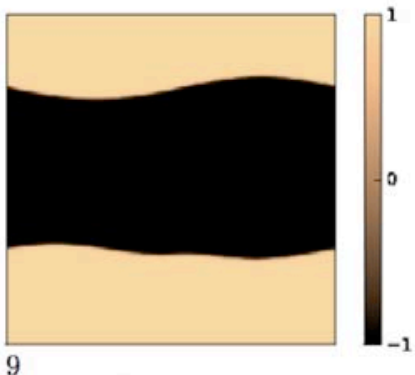

9

Figure 2: Typical long-term patterns of the distribution of solid and liquid regions resulting from the simulation of the local model (top) and the nonlocal model (bottom) run to what appear to be stationary solutions. Here, the local model has achieved a simple geometry characterised by the minimal length of the solid/liquid boundary. While not shown here, another minimal configuration in two dimensions would correspond to circular interfaces [9]. The nonlocal model appears to stop coarsening and maintain meandering interfaces.

while the nonlocal model (4) maintains meandering interfaces throughout the entirety of the simulations. While it is beyond the scope of this paper, it should be noted that long term-solutions to isothermal formulations of (4) and (5) have been studied analytically. One goal has been to describe the geometry of the patterns formed by the equilibrium solutions of these evolution equations. While the isothermal version of the local Allen-Cahn equation in (5) is known to have a compact attractor and only settle into simple geometries, the isothermal version of the nonlocal extension in (4) does not have a compact attractor, and is shown to stop coarsening, which implies that stable solutions will have more complicated geometries [5]. The numerical investigations here operate on non-isothermal systems, which lie outside the necessary assumptions for the results obtained in [5], however the numerical investigations here and the more thorough investigations carried out in [9] indicate that the fundamental differences between the stability results in [5] may carry over into these coupled systems.

\section{Numerical Simulation}

The numerical implementation of these models has been derived from a spectral method known as the Galerkin Method. This method takes advantage of the periodic boundary conditions as well as transforming the computation of the convolution into the direct multiplication of Fourier coefficients followed by an Inverse Discrete Fourier Transform. 
Several other implementations are considered in [8], and the Galerkin Method is determined to be superior due to the exactness of the spatial derivatives of $u(t, x)$ and $\phi(t, x)$, as well as from the efficiency of the computation of $J * \phi$. The following discussion closely follows [8] $§ 2.4,2.6$.

The spectral approach to approximating solutions to differential equations begins by considering the expansion of the unknown functions into their respective linear combinations of orthogonal basis functions. Here we consider traditional Fourier series, and as an example we take the Nonlocal Model in (4) in one spatial dimension for brevity, revealing

$$
u^{N}(t, x)=\sum_{j=-\frac{N}{2}-1}^{\frac{N}{2}} C_{j}(t) e^{2 \pi i j x} \quad \text { and } \quad \phi^{N}(t, x)=\sum_{j=-\frac{N}{2}-1}^{\frac{N}{2}} D_{j}(t) e^{2 \pi i j x},
$$

where the superscript $N$ indicates that these are finite approximations. Noting that the convolution term $J * \phi=\int_{0}^{1} J(x-y) \phi(x) d y$, we take its expansion as well. With $J$ expressed as

$$
J^{N}(t, x)=\sum_{j=-\frac{N}{2}-1}^{\frac{N}{2}} J_{j}(t) e^{2 \pi i j x}
$$

we can write the convolution as

$$
J^{N}(x) * \phi^{N}(t, x)=\int_{0}^{1}\left[\left(\sum_{j=-\frac{N}{2}+1}^{\frac{N}{2}} J_{j}(t) e^{2 \pi i j x-2 \pi i j y}\right)\left(\sum_{k=-\frac{N}{2}-1}^{\frac{N}{2}} D_{k}(t) e^{2 \pi i k x}\right)\right] d y .
$$

At this point a consideration must be made for the nonlinearity $\phi^{3}$ that appears in (6). Simply cubing the finite approximation to $\phi$ will result in higher-order terms in the Fourier Series with nonzero coefficients. To handle the nonlinearity we use the information from an $N$-tuple of already known values for $\phi$ coming from the initial condition, or from the previous time step in the computation. Applying the Inverse Fourier Transform will produce coefficients for an $N$-term approximation to $\phi^{3}$. We will represent this approximation to $\phi^{3}(t, x)$ as

$$
\phi^{3}(t, x) \approx \xi^{N}(t, x)=\sum_{j=-\frac{N}{2}-1}^{\frac{N}{2}} \xi_{j}(t) e^{2 \pi i j x}
$$

and now the pieces are in place to express the model in a form amenable to numerical implementation.

The system in (4) can be tentatively expressed as

$$
\begin{aligned}
u_{t}^{N} & \doteq \Delta u^{N}-\lambda \phi_{t}^{N} \\
\varepsilon^{2} \phi_{t}^{N} & \doteq J^{N} * \phi^{N}-c \phi^{N}+\phi^{N}-\xi^{N}+u^{N}
\end{aligned}
$$

where the dots above the 'equal' signs indicate that we will not have true equality here. The truncation of the Fourier series has forced the numerical solutions $u$ and $\phi$ from (4) to lie in a finite-dimensional space, and the nonlinear terms $J * \phi$ and $\phi^{3}$ disallow trivial equality of the Fourier coefficients in this finite representation.

By allowing the solutions to come from an $N$-dimensional space spanned by orthogonal basis functions, again of the form $e^{2 \pi i l x}$, with $-\frac{N}{2}+1 \leq l \leq \frac{N}{2}$, we can require that the 
residuals $\left(u_{t}^{N}-\Delta u^{N}+\lambda \phi_{t}^{N}\right)$ and $\left(\varepsilon^{2} \phi_{t}^{N}-J^{N} * \phi^{N}+c \phi^{N}-\phi^{N}+\xi^{N}-u^{N}\right)$ be orthogonal to the solution subspace, which for example looks like

$$
\int_{0}^{1}\left[\sum_{j=-\frac{N}{2}-1}^{\frac{N}{2}}\left(\frac{d}{d t} C_{j}(t)+4 \pi^{2} j^{2} \frac{d}{d t} C_{j}(t)+\lambda \frac{d}{d t} D_{j}(t)\right) e^{2 \pi i j x}\right] e^{2 \pi i l x} d x=0 .
$$

The distance from the residuals to the solution space will be just the magnitude of the Fourier coefficients, which suggests that this orthogonality condition will lead to a very good approximation.

The orthogonality condition turns out to collapse the complicated system into a set of ordinary differential equations in terms of the Fourier coefficients $\hat{u}, \hat{\phi}, \hat{J}$, and $\hat{\xi}$ in time. In our simulations (which, along with a fuller description and analysis, were developed by the work of Hartley in [8]) the set of equations are solved forward in time using the central difference formula $\left(\frac{\partial f}{\partial t}\right)_{k} \approx \frac{f_{k+1}-f_{k}}{\Delta t}$. This setup allows for the solution to all linear terms to be implicit, and requires the explicit solution only for the single nonlinear term. Using the superscript $j$ to denote the spatial coordinate and the subscript $k$ to express the time step, we have the following equations in Fourier coefficients.

$$
\begin{gathered}
\frac{\hat{u}_{k+1}^{j}-\hat{u}_{k}^{j}}{\Delta t}=4 \pi^{2} j^{2} \hat{u}_{k+1}^{j}-\lambda \frac{\hat{\phi}_{k+1}^{j}-\hat{\phi}_{k}^{j}}{\Delta t} \\
\varepsilon^{2} \frac{\hat{\phi}_{k+1}^{j}-\hat{\phi}_{k}^{j}}{\Delta t}=\hat{J}_{k+1}^{j} \hat{\phi}_{k+1}^{j}+\hat{\phi}_{k+1}^{j}-\hat{\xi}_{k}^{j}-\hat{u}_{k+1}^{j}
\end{gathered}
$$

\section{Pattern Formation}

The patterns we are interested in correspond to overhead images of the entire domain, revealing the distribution of liquid and solid regions in the sample. This amounts to a plot of the phase-field variable at a fixed time step, which can be viewed as a map from the square $\Omega \subseteq \mathbb{R}^{2}$ into $\mathbb{R}$. Obtaining an image of the pattern is achieved by assigning a color scheme to represent the phase field values as they range between -1 and +1 , and plotting $\phi$ on the two-dimensional square domain. The model includes the full description of the temperature field at each time step as well, and this can be plotted in a similar manner. Plots of the temperature field variable reveal 'hot' (or 'cold') spots on the domain, and our analysis will investigate the relationship between these occurrences and fundamental changes to the pattern of the phase as the models evolve toward equilibrium.

The initial conditions place the domain into a well-mixed state, consisting of a random distribution of tiny regions existing as not quite solid or liquid. Thus the starting pattern takes on colors close to the middle ground between the liquid and solid indicators, and is uninteresting outside of the verification that we begin with a well-mixed condition. Within several time steps the double-well potential function exerts its influence and begins to decompose the domain into regions of primarily one phase or another. We see this behavior played out in a snapshot of the phase-field variable by the emergence of a finely structured pattern of very small regions of liquid and solid (refer to Figure 4). That these regions are actually being drawn toward the values -1 and +1 can be seen in the plot of the infinity norm of $\phi$ taken over time (see Figure 3).

The pattern in the phase field evolves toward a geometry that corresponds to a nearequilibrium solution to the minimization of the free energy formulation specific to the 


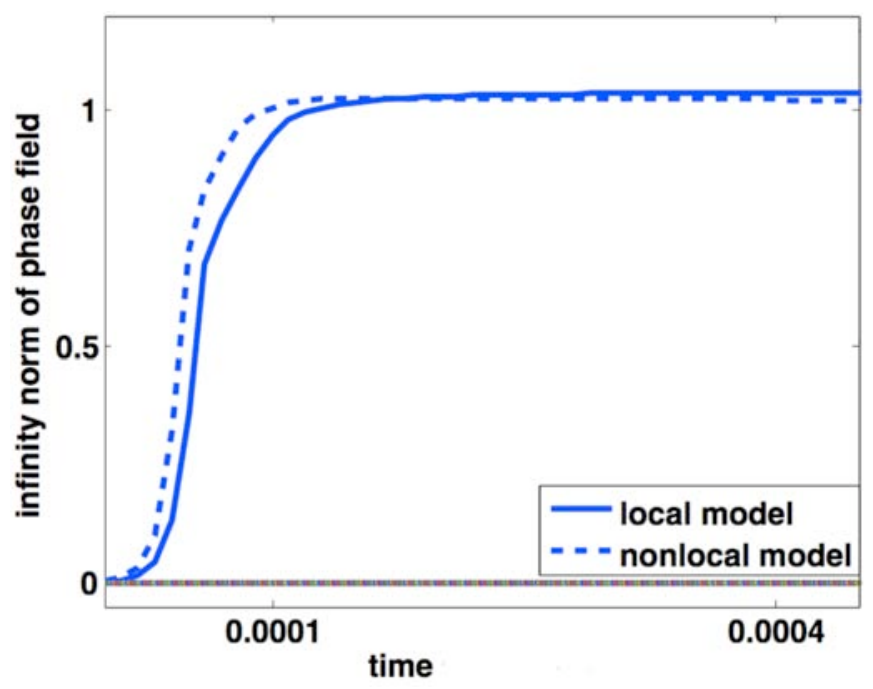

Figure 3: Early phase decomposition is inferred from the steep increase in the value of the infinity norm of the phase-field variable $\phi$ with respect to time. At time zero the domain exists at an unstable location along the potential energy curve in Figure 1, and is seen to quickly decompose into regions that are approximately solid or liquid as the total free energy of the system is decreased. An alternative illustration of the decomposition of the domain can be seen by the successive plots of $\phi$ early in the simulation, shown in Figure 4 .

model. The result of this minimization coarsens the pattern, enlarging regions of liquid or solid and creating odd-shaped patches in the pattern. Occasionally these patches merge, and occasionally this merge completely traps a region of opposite composition. On the way to minimizing the total length of interfaces, this trapped region decreases in size and its boundary takes the shape of a circle. Eventually the region is diminished to a size that requires a very sharp interface, at which point the model forces the value of the phase-field variable to move across the energy barrier traced out by the potential function $F$, thus rapidly solidifying or liquifying the small region. In this way, the many connected regions are destroyed one-by-one, coarsening the pattern in the phase field.

The variations in the temperature field are encoded in $u(t, x)$, and have been considered against the pattern development occurring in $\phi(t, x)$. In Figure 5 we see that the temperature field maintains a shadow of the pattern in the phase field over time. What else is noticed is that during periods corresponding to smooth deformations of the phase field, we observe only slight variations in the underlying temperature field throughout the domain. On the other hand as the phase field evolves to completely destroy a connected region of either liquid or solid, the temperature field reflects this activity with a significant burst of energy occurring precisely in the region being destroyed.

This behavior is more clearly illustrated in Figure 6. A plot of the infinity norm of $u$ is shown for a short time period, the information being captured in this measure is the maximum fluctuation in the temperature field achieved on the square domain. It can be seen from (4) that significant changes in $\phi_{t}$ will result in sizeable fluctuations in $u_{t}$. What is lost is any indication of where the maximum of these fluctuations occurs on the square. Taking a look at the pattern over the same duration we can see that the regions in the temperature field corresponding to the spikes in $\|u\|_{\infty}$ are precisely the regions in the phase field where fundamental changes to the pattern are occurring. 

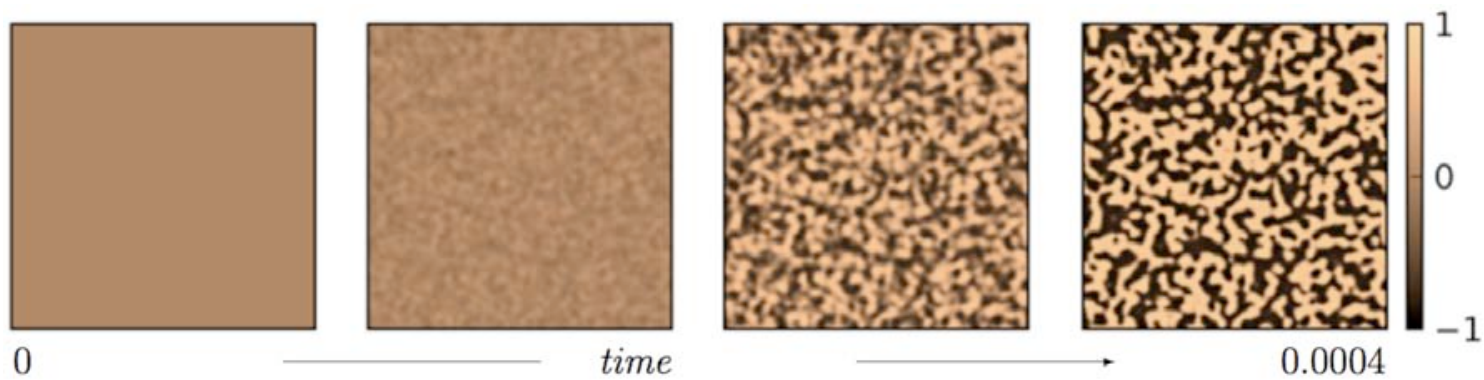

Figure 4: Early phase decomposition beginning from an initial condition randomized as small values around zero. The domain is transitioning to solid in the dark regions, and to liquid in the light regions. These snapshots were taken during the first forty time steps under the nonlocal model, $\alpha=1$. An alternative illustration of the decomposition of the domain can be seen in the plot of the infinity norm of $\phi$ over time in Figure 3
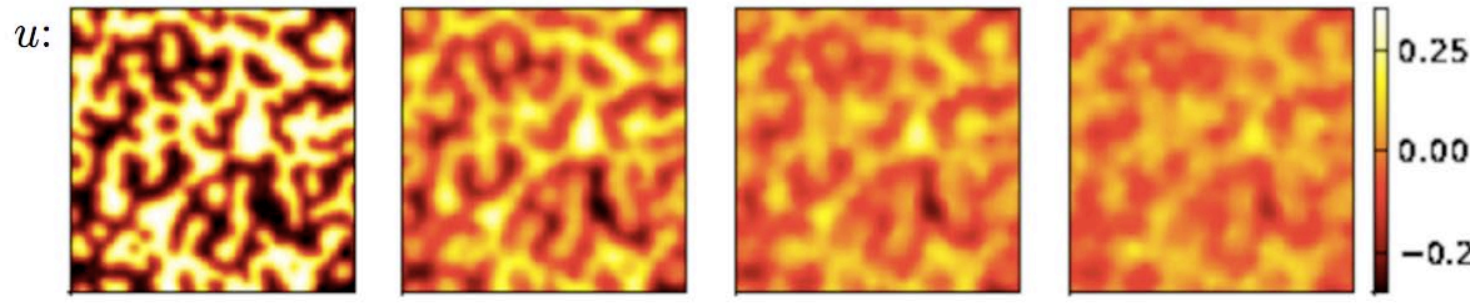

$\phi:$
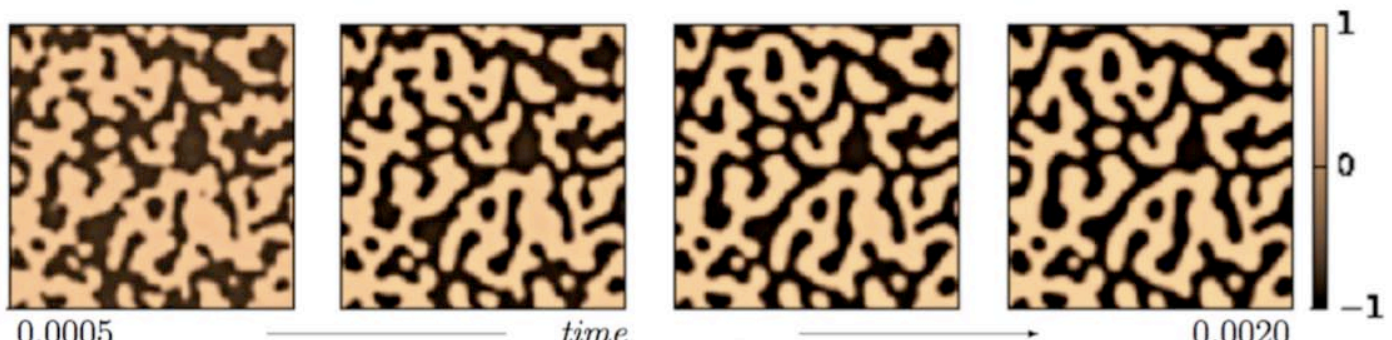

Figure 5: The underlying temperature field $u$ (top series) is seen to correspond to the patterns in the phase field $\phi$ (bottom series). The image shows the decomposition of the domain into distinctly solid and liquid regions accompanied by closely related activity in the underlying temperature field. Material that is solidifying, represented by the dark regions of $\phi$, are seen to be releasing thermal energy into the underlying temperature field, indicated by bright regions in $u$. Similarly, liquifying regions of the phase field are seen to be absorbing thermal energy from the underlying temperature field. 


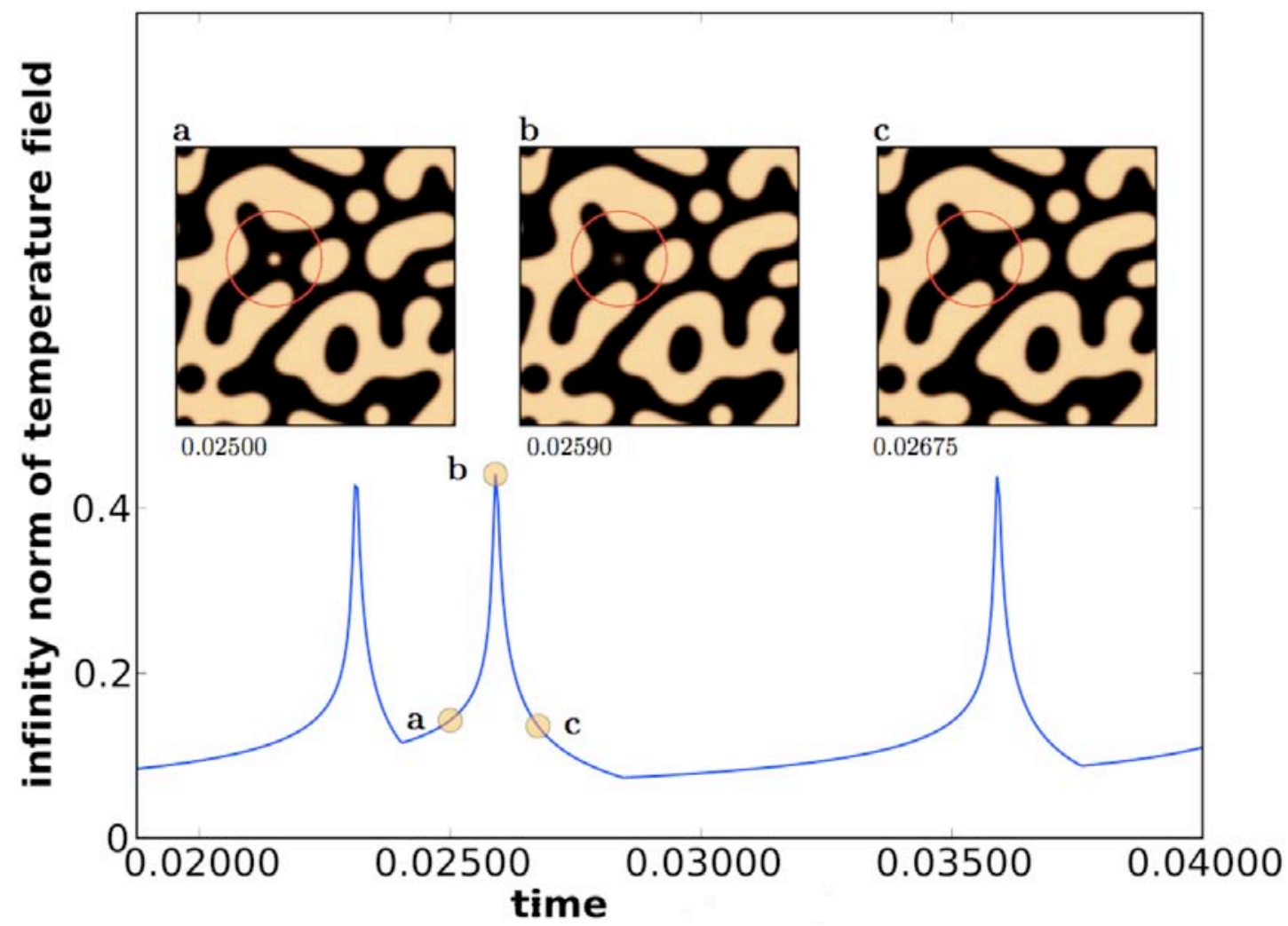

Figure 6: Destruction of connected regions coincide with bursts occurring in the temperature field. The infinity norm of the underlying temperature field corresponding to the images $\mathbf{a}, \mathbf{b}$, and $\mathbf{c}$ lie along the graph as marked by their corresponding circles. The left and right-most spikes indicate similar events in the phase field, however the location of these occurrences on the square is impossible to deduce from this measure. The sharp corners in the valleys of $\|u\|_{\infty}$ are the result of this loss of information, the corners indicate the level where one diminishing spike is passed by a rising spike elsewhere on the domain. 


\section{Analysis of Patterns}

In order to investigate the differences between our two models, following [8], we consider a hybrid form of (4) and (5) by forming their convex combination, parameterized by the letter $\alpha$ as shown in (9). We have investigated early pattern formation under this model for values of $\alpha \in\{0,0.2,0.4,0.6,0.8,1.0\}$. As the initial conditions have been randomized, we cannot expect a single simulation to provide a complete picture of early pattern development for any particular value of $\alpha$. Rather, we have considered the average behavior as determined over one thousand simulations for each value of $\alpha$.

\section{Hybrid Model}

$$
\begin{aligned}
u_{t} & =\Delta u-\lambda \phi_{t}, \\
\varepsilon^{2} \phi_{t} & =(1-\alpha) \varepsilon^{2} \Delta \phi+\alpha(J * \phi-c \phi)+f(\phi, u)
\end{aligned}
$$

In the previous section it was pointed out that certain changes in pattern complexity were marked by corresponding activity in the temperature field. With one thousand simulations at each parameter value we are able to demonstrate a difference in the rate of the occurrence of the temperature bursts with respect to $\alpha$. Figure 7 plots a count of the significant spikes in the infinity norm of $u$ during an early stage in the pattern evolution ${ }^{1}$. We see that for the purely local model (5) connected regions persist in the phase field, whereas in the nonlocal model (4) more connected regions are destroyed in the same time period. Accompanying this plot is Figure 8, where the graphs of the peak counts over time have been numerically differentiated in order to compare rates of peaking behavior in the same time period. The intuition that the local model is somehow maintaining its pattern complexity longer is supported by the observation of the temperature field bursts occurring at a slower rate than what is seen in the nonlocal model.

\section{Computational homology}

The gross features of the pattern in the phase field appear similar under both models. The patterns are also rather complex, so visual examinations of the results alone are inadequate to identify the more subtle features relating to physical properties of the materials we are approximating. In order to address these concerns we have employed the technique of computational homology to produce quantitative descriptions of the patterns in the phase

\footnotetext{
1

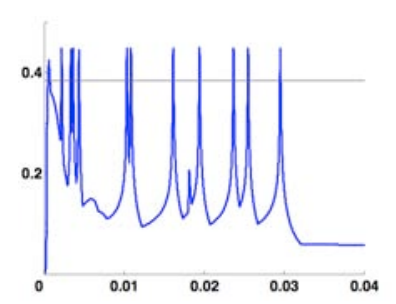

For each initial condition we can record the largest value in the temperature field occurring at each time step during the simulation, this is $\|u\|_{\infty}$ vs. time. The accompanying figure is an example of such a plot. In order to automate the peak counting, we consider a threshold value for $\|u\|_{\infty}$, appearing here as a horizontal line at eighty-five percent of the maximum peak height. The peaks are counted by considering the number of times the graph passes through this threshold, interpreting two crossings as a single peak - the first cross identifies the positively sloping portion of the peak and the second cross identifies the negatively sloping portion of the peak. A weakness of this method is that peaks in the graph that do not achieve this height are disregarded. This weakness was overshadowed by the desire to capture peaks occurring in rapid succession early in the evolution of the phase. For instance, consider the infinity norm tracking nearly simultaneous bursts. It is seen early in the accompanying plot that the infinity norm does not necessarily descend too far below the maximum peak value before another significant peak occurs. Lowering the threshold introduces the possibility that several peaks occurring in rapid succession will be counted as a single one. For our purposes, this is highly undesirable.
} 
field. (The technique in this setting has been introduced in the work of Hartley [8] and Wanner et al. [7]).

At any point in time a snapshot of the phase field can be partitioned by classifying points taking values near -1 as equivalent, and points near +1 as being equivalent. This simplifies the domain and enables us to speak about regions as either entirely solid or liquid. We are then able to ask topological questions about this partition. Specifically, we are able to resolve the homology groups on one of the constructed equivalence classes in the domain and count the Betti numbers of dimension zero and dimension one. The dimension zero Betti number gives a count of the number of connected regions in the equivalence class. Our model displays symmetric equivalence classes and this discussion will refer to the class that corresponds to the solid phase. A connected region on the two dimensional domain is a set that contains a path that can reach any point without leaving the set. This would be an uninterrupted solid patch, which can take any shape and can even wrap around the domain (in accordance with the periodic boundary conditions). The dimension one Betti number gives a count of the number of holes in the pattern, this occurs when the set has enclosed a portion of its complement. The dimension one Betti number counts the number of patches of liquid that have been completely surrounded by a solid region. Computing these numbers recasts our focus on changes in the pattern into geometrical vocabulary. As well, it allows for the investigation of pattern evolution separated from any attention to the underlying temperature field.

Figures 9 and 10 are plots of the dimension zero and dimension one Betti numbers computed for the patterns in the phase field. The similarity is a consequence of the symmetry between solid and liquid regions in our particular model. What is noticed in these plots is the monotonicity of the decrease in pattern complexity over time. From this behavior we see that the weighted (or unweighted, local) contribution to the free energy from the existence of interfaces, along with the double-well potential function conspire to forbid spontaneous transitions from one phase of matter to the other. As with the data from the temperature field, the homology analysis shows persistence of complexity in the pattern in the local model. We also see the same gradual shift in these graphs as the parameter $\alpha$ is decreased to 0 .

\section{Conclusions}

The nonlocal extension to the classical phase-field model relies on a weighted averaging of a larger portion of an interface than is considered by the gradient operator of the classical model. From a phenomenological perspective, this more fully accounts for the mechanisms of diffuse-interface phenomena as originally proposed by van der Waals over one hundred years ago. Furthermore, the system of partial differential equations derived from the nonlocal model of the free energy are less well understood than those coming from the gradient formulation. By paying close attention to the interplay between the two phase-field variables $u$ and $\phi$, which are the coupled objects of our models, we hope to have revealed some of the connection between what can be interpreted as an underlying temperature field $u(t, x)$ and an order parameter $\phi(t, x)$.

The computational homology results provide a means of comparing patterns in the phase field for randomized initial conditions, as well as providing a comparison of the results of our two models. These comparisons are achieved by considering the number of connected sets in a given pattern, revealing the extent to which the two phases have separated from one another and how 'complicated' the pattern has become. The nonlocal model was shown 


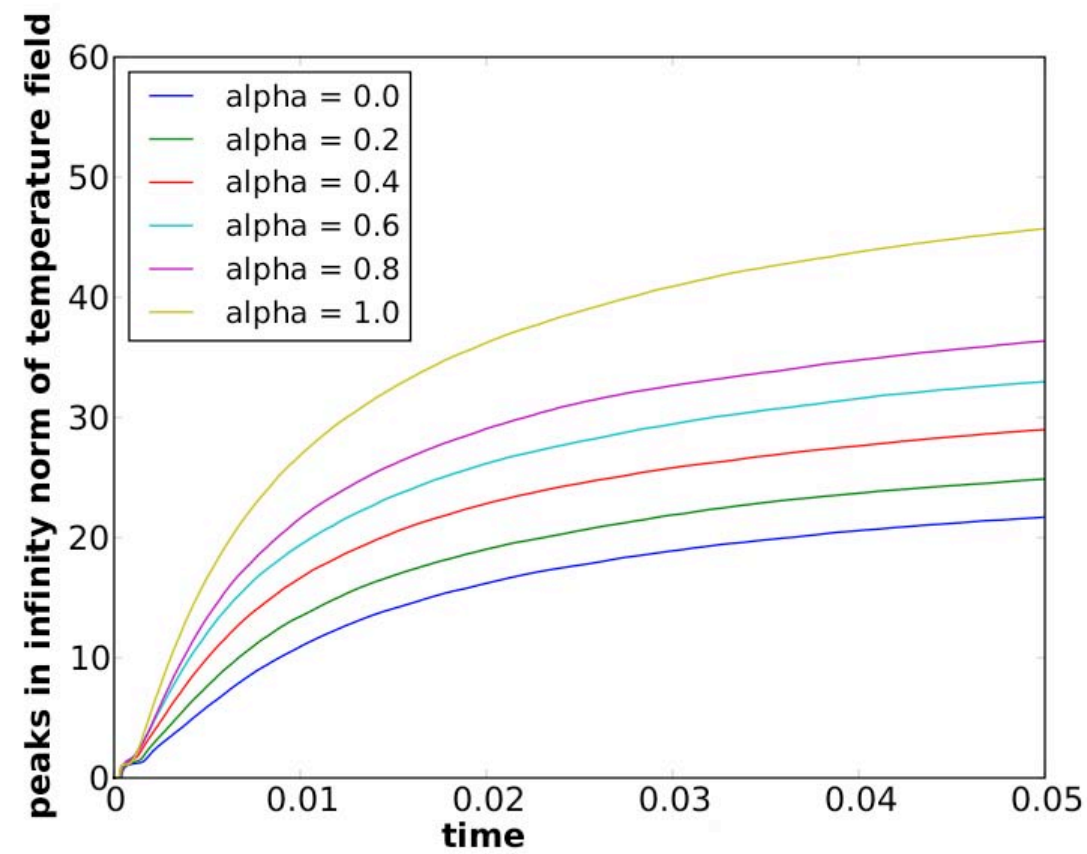

Figure 7: A count of the quantity of significant peaks in the temperature field over time. The curves are the result of averaging one thousand simulations for each value of $\alpha$. Peaking appears to occur early in the simulation. The derivative of these curves is shown in Figure 8.

to maintain a larger quantity of disconnected regions during the solidification process, and appears to approach a similar pattern as the local model over the very long run. This implies that phase separation is taking longer to occur under the nonlocal regime.

The underlying temperature field is noticed to be correlated to the phase field during the solidification process. We observe a strong and rapid response in the temperature field a regions of one phase are diminished and transformed into the opposite phase (see Figure 6). As may be expected, these events very closely follow a decrease in the complexity of the pattern as indicated by the associated decrease in the Betti Number associated with the relevant phase.

\section{Acknowledgements}

The research presented here investigates an aspect of the results on non-isothermal phase separation presented by Tina Hartley in her doctoral thesis at George Mason University [8]. The computational homology work was performed using the free and open source CHomP software (available here: http://chomp.rutgers.edu/). I would like to thank the anonymous referees for their suggestions and for their time. This work was supported by the CSUMS Grant for Undergraduate Research in Computational Mathematics at George Mason University, DMS-0639300. 


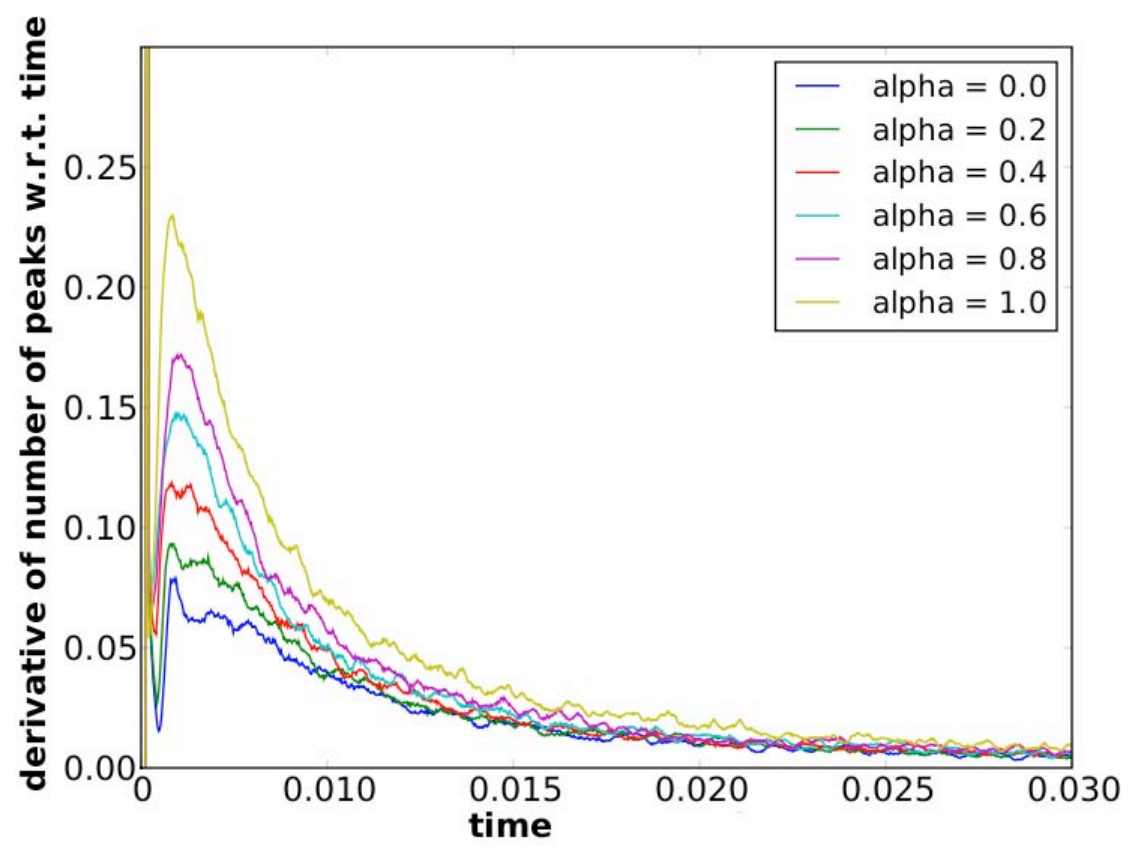

Figure 8: Numerical derivatives of the curves in Figure 7 The graphs indicate the rate of bursts occurring in the temperature field early in the pattern evolution. The initial curves were smoothed using a Gaussian weighting function before a central difference formula was applied to the data. 


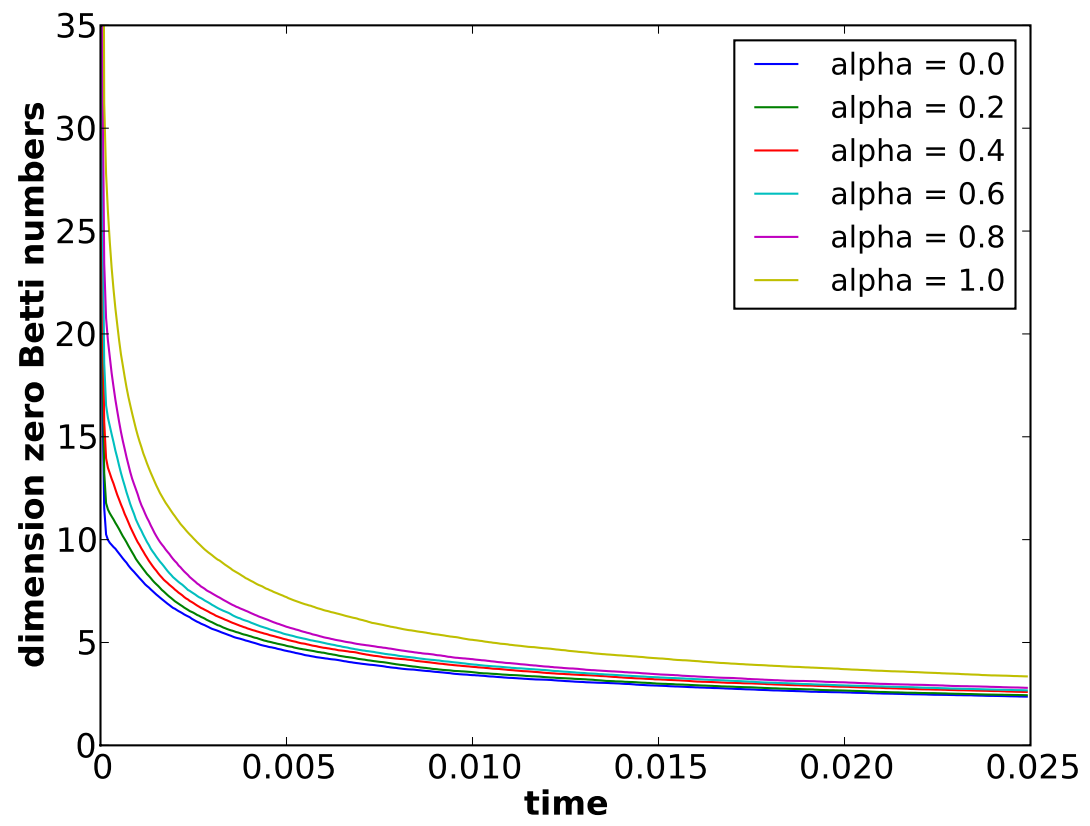

Figure 9: Dimension zero Betti numbers computed for several values of $\alpha$. This is a count of the number of connected solid regions in the pattern of the phase field over time, and has been determined by averaging the behavior of one thousand simulations for each value of $\alpha$. The purely nonlocal model from (2) is represented in the top curve, and the purely local model (3) is represented in the bottom curve. 


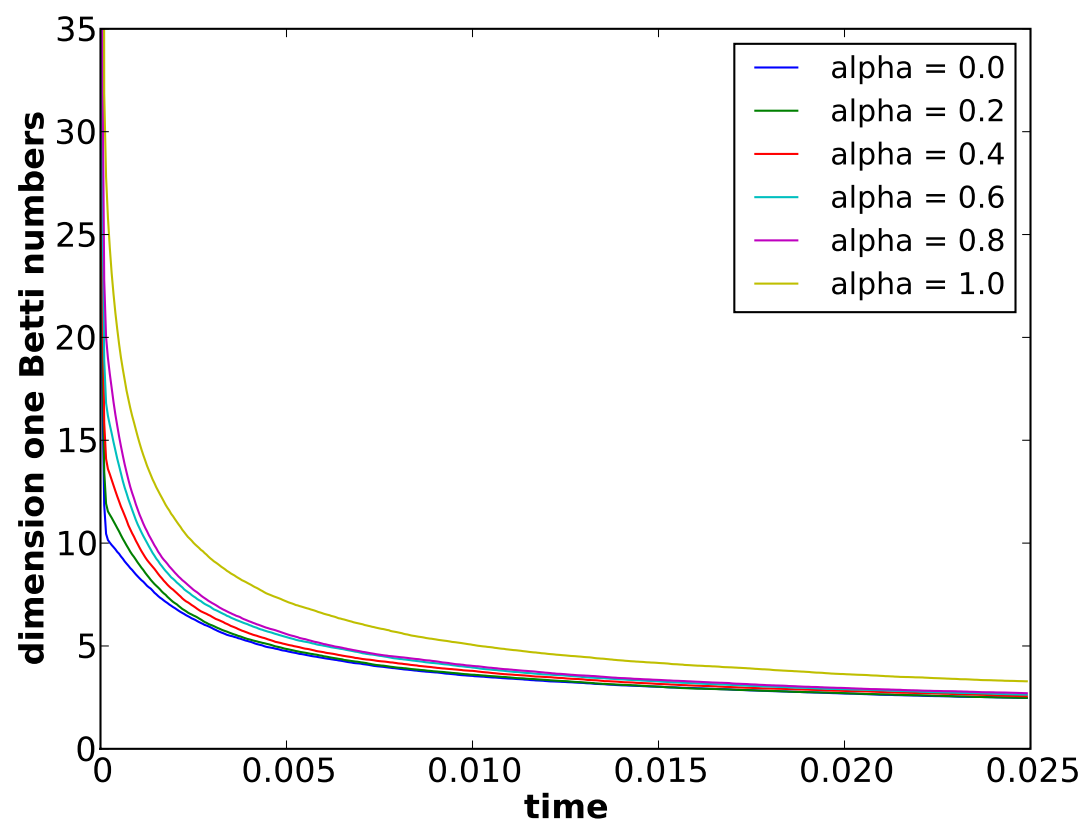

Figure 10: Dimension one Betti numbers computed for several values of $\alpha$. This is a count of the number of holes in the solid regions in the pattern of the phase field over time and for this particular model corresponds to the number of connected liquid regions. The curves have been determined by averaging the behavior of one thousand simulations for each value of $\alpha$. The purely nonlocal model from (2) is represented in the top curve, and the purely local model (3) is represented in the bottom curve. 


\section{References}

[1] W. J. Boettinger, J. A. Warren, C. Bekermann, and A. Karma. Phase-field simulation of solidification. Annual Review of Materials Research, 32:pp. 163-194, 2002.

[2] John W. Cahn. Free energy of a nonuniform system II. Thermodynamic basis. The Journal of Chemical Physics, Vol. 30(5), 1959.

[3] John W. Cahn. Theory of crystal growth and interface motion in crystalline materials. Acta Metallurgica, Vol. 8, 1960.

[4] John W. Cahn and John E. Hilliard. Free energy of a nonuniform system I. Interfacial free energy. The Journal of Chemical Physics, Vol. 28(2), 1958.

[5] Dugald B. Duncan, Micheal Grinfeld, and Iulian Stoleriu. Coarsening in an integrodifferential model of phase transitions. European Journal of Applied Mathematics, Vol. 11:pp. 561-572, 2000.

[6] Paul C. Fife. Models for phase separation and their mathematics. Electronic Journal of Differential Equations, Vol. 2000(48):pp. 1-26, 2000.

[7] Marcio Gameiro, Konstantin Mischaikow, and Thomas Wanner. Evolution of pattern complexity in the Cahn-Hilliard theory of phase separation. Acta Materialia, Vol. 53(3):pp. 693-704, 2005.

[8] Tina Hartley. An Anlysis of Phase Separation Processes for Stochastic and Nonlocal Extensions of the Classical Phase-Field Model. PhD thesis, George Mason University, 2008.

[9] Tina Hartley and Thomas Wanner. A semi-implicit spectral method for stochastic nonlocal phase-field models. Discrete and Continuous Dynamical Systems, Series A, Vol. 25(2):pp. 399-429, 2009. 